\title{
Thermal orientation of electron spins
}

\author{
S. A. Tarasenko \\ A.F. Ioffe Physico-Technical Institute, Russian Academy of Sciences, 194021 St. Petersburg, Russia
}

\begin{abstract}
It is shown that the spin orientation of free electrons occurs in low-symmetry semiconductor structures if only the electron gas is driven out of thermal equilibrium with the crystal lattice. The proposed mechanism of such a thermal orientation of electron spins is based on spin dependence of the electron-phonon interaction which tends to restore equilibrium. The microscopic theory of the effect is developed here for asymmetric (110)-grown quantum wells where the electron gas heating leads to the spin orientation along the [110] axis in the quantum well plane.
\end{abstract}

PACS numbers: 72.25.Rb, 73.63.Hs, 75.75.+a

\section{INTRODUCTION}

The spin-related phenomena in semiconductors have been attracting considerable attention since the discovery of the optical orientation of electron and nuclear spins and the basic mechanisms of spin relaxation [1]. Much effort in this field is currently focused on the development of novel methods of spin orientation of free carriers ranging from the spin Hall effect [2] and spin-dependent tunneling 3] to the optical orientation by linearly polarized light [4]. Here we show that the spin orientation of free electrons can be achieved in semiconductor nanostructures by simple electron gas heating. We demonstrate that, in nanostructures of sufficiently low space symmetry, the electron-phonon interaction tending to restore equilibrium between the electrons and the crystal lattice is spin-dependent and leads to the spin orientation of carriers. Such a thermal orientation of electron spins is considered here for asymmetric (110)-grown quantum wells (QWs) where the electron gas heating leads to the spin orientation along the [1]0] axis in the QW plane.

The possibility to achieve the spin polarization of carriers $\mathbf{S}$ caused by the disturbance of thermal equilibrium in asymmetric (110)-grown QWs follows from symmetry analysis. Indeed, such structures belong to the point group $C_{s}$ that contains only two symmetry elements, namely, identity and a mirror plane perpendicular to the $x$ axis. Here we use the following coordinate frame: $x \|[1 \overline{1} 0]$ and $y \|[00 \overline{1}]$ are the in-plane axes, and $z \|[110]$ is the growth direction. Reflection by the mirror plane changes the sign of the $y$ and $z$ components of the spin axial vector $\mathbf{S}$ but does not modify the $x$ component. Therefore, the spin component $S_{x}$ is an invariant in asymmetric (110)-grown QWs suggesting that the spin polarization along the $x$ axis can emerge if the electron gas is driven out of equilibrium by any means. Particularly, in the case of disturbance of thermal equilibrium between the electrons and the crystal lattice, the spin orientation can phenomenologically be described by

$$
S_{x} \propto \frac{\Delta T}{T_{e}},
$$

where $\Delta T=T_{e}-T_{0}, T_{e}$ and $T_{0}$ are the electron and lattice temperatures, respectively.

\section{MICROSCOPIC MODEL}

The microscopic model of the thermal orientation of electron spins is caused by the energy relaxation of hot carriers and includes two stages which are illustrated in Fig. 1(a) and Fig. 1(b).

In the first stage [Fig. 1(a)], the carriers lose a part of their kinetic energy by emitting phonons. Such energy relaxation processes shown by curved arrows are spin-dependent [5]. In QW structures without an inversion center, spin-orbit interaction adds an asymmetric term to the probability of electron scattering by phonons which is linear in the wave vector components [6]. As is shown in the next Section, the dominant spin-dependent contribution to the probability of electron scattering in (110)-grown QWs is proportional to $\sigma_{z}\left(k_{x}+k_{x}^{\prime}\right)$, where $\sigma_{z}$ is the Pauli matrix, $k_{x}$ and $k_{x}^{\prime}$ are components of the initial and scattered electron wave vectors. Due to the spin-dependent asymmetry of the electron-phonon interaction, electrons with the spin "up" (along the $z$ axis) predominantly vacate the excited states with positive $k_{x}$ while electrons with the spin "down" vacate the excited states with negative $k_{x}$. This leads to a nonequilibrium distribution where the spin-up hot carriers occupy mainly the left-hand branch of the dispersion curve (carriers with the opposite spin orientation have gone to the subband
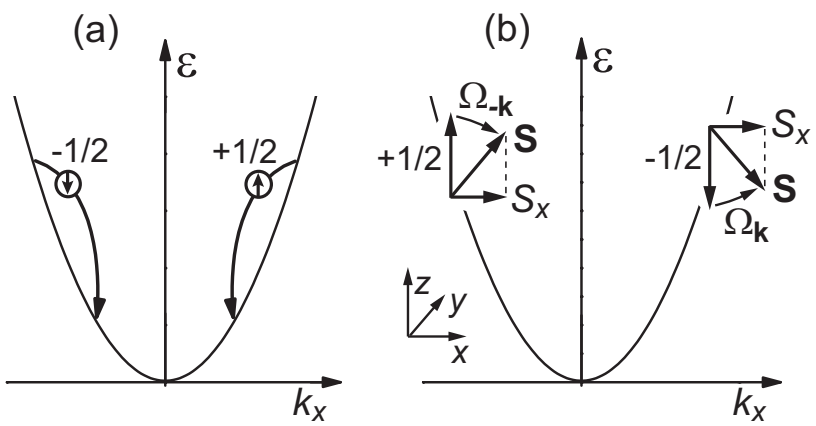

FIG. 1: Microscopic origin of the electron gas spin orientation due to the energy relaxation of hot carriers. (a) Spindependent asymmetry of the electron-phonon interaction followed by (b) spin precession in the effective magnetic field leads to the spin orientation of electrons along the $x$ axis. 
bottom) while the spin-down carriers occupy mainly the right-hand branch.

In the second stage [Fig. 1(b)], a net spin orientation of the electron gas appears as a result of the subsequent spin precession of nonequilibrium carriers in the effective magnetic field induced by the spin-orbit interaction [7]. The field has nonzero in-plane components in asymmetrically growth QWs, e.g., due to the Rashba effect [8]. Therefore, the spins of nonequilibrium carriers, directed along or opposite to the $z$ axis after the phonon emission, will precess in the effective field as shown in Fig. 1(b). Note that electrons with the initial spin $-1 / 2$ and wave vector $k_{x}>0$ are acted upon by the effective field with the Larmor frequency $\boldsymbol{\Omega}_{\mathbf{k}}$, while particles with the initial spin $+1 / 2$ and the negative wave vector feel the field with the Larmor frequency $\boldsymbol{\Omega}_{-\mathbf{k}}$. The effective magnetic field induced by spin-orbit interaction is an odd function of the wave vector, therefore, $\boldsymbol{\Omega}_{-\mathbf{k}}=-\boldsymbol{\Omega}_{\mathbf{k}}$, and the spins of particles with positive and negative values of $k_{x}$ rotate in opposite directions. This gives rise to the spin component $S_{x}>0$ for all hot electrons in the subband and the net spin polarization of the electron gas.

We note that the electron spin caused by the energy relaxation processes is nonequilibrium and, therefore, can be rotated by an external static magnetic field, similarly to the Hanle effect in optics.

\section{THEORY}

The spin-dependent asymmetry of the electron-phonon interaction can be obtained if one takes into account both $\mathbf{k} \cdot \mathbf{p}$ admixture of the valence-band states to the conduction-band wave function and the phonon-induced interband coupling. To first order in the $\mathbf{k} \cdot \mathbf{p}$ theory, where $\mathbf{k}=\left(k_{x}, k_{y}\right)$ is the in-plane wave vector, the electron wave function in a (110)-grown QW has the form

$$
\Psi_{\mathbf{k}}(\mathbf{r})=S \psi+X^{\prime} \frac{v_{x}+v_{z}}{\sqrt{2}}+Y^{\prime} \frac{v_{z}-v_{x}}{\sqrt{2}}-Z^{\prime} v_{y} .
$$

Here $S$ and $X^{\prime}, Y^{\prime}, Z^{\prime}$ are the Bloch functions of the conduction and valence bands at the $\Gamma$ point of the Brillouin zone, $\psi$ and $\mathbf{v}=\left(v_{x}, v_{y}, v_{z}\right)$ are the envelope spinors. The Bloch functions $X^{\prime}, Y^{\prime}$, and $Z^{\prime}$ in Eq. (2) are referred to the cubic axes $x^{\prime}\left\|[100], y^{\prime}\right\|[010]$, and $z^{\prime} \|[001]$, respectively. The envelope spinors of the valence-band states are related to the conduction-band spinor by

$$
\mathbf{v}=-\frac{\hbar P_{c v}}{3 m_{0}} \frac{\left(3 E_{g}+2 \Delta_{s o}\right) \mathbf{k}+i \Delta_{s o}[\sigma \times \mathbf{k}]}{E_{g}\left(E_{g}+\Delta_{s o}\right)} \psi,
$$

where $P_{c v}=\left\langle S\left|p_{z^{\prime}}\right| Z^{\prime}\right\rangle$ is the interband matrix element of the momentum operator, $m_{0}$ is the free electron mass, $E_{g}$ is the band gap, $\Delta_{s o}$ is the valence-band spin-orbit splitting, and $\sigma$ is the vector of the Pauli matrices.

We consider the electron scattering by acoustic phonons due to the deformation-potential mechanism. In cubic noncentrosymmetric crystals such as zinc-blendetype semiconductors, the strain induces a coupling between the conduction-band and valence-band states 1, 9]. The matrix elements of such a coupling have the form $V_{S, X^{\prime}}=\Xi_{c v} u_{y^{\prime} z^{\prime}}, V_{S, Y^{\prime}}=\Xi_{c v} u_{x^{\prime} z^{\prime}}, V_{S, Z^{\prime}}=\Xi_{c v} u_{x^{\prime} y^{\prime}}$, where $\Xi_{c v}$ is the interband constant of the deformation potential, and $u_{\alpha \beta}$ are the strain tensor components used here in the primed coordinate system. It is the straininduced interband coupling together with the spin-orbit splitting of the valence band that leads to spin-dependent asymmetry of the electron-phonon interaction. Taking into account $\mathbf{k} \cdot \mathbf{p}$ mixing given by Eqs. (2) and (3) and allowing for the interband coupling, we derive for the Hamiltonian of the electron-phonon interaction in (110)grown quantum wells

$$
\begin{gathered}
V_{\mathrm{el}-\mathrm{phon}}\left(\mathbf{k}^{\prime}, \mathbf{k}\right)=\Xi_{c} \sum_{\alpha} u_{\alpha \alpha}+\xi \Xi_{c v}\left\{\left(k_{x}+k_{x}^{\prime}\right) \times\right. \\
\left.\left[\sigma_{z}\left(u_{z z}-u_{x x}\right) / 2-\sigma_{y} u_{y z}\right]+\left(k_{y}+k_{y}^{\prime}\right)\left[\sigma_{x} u_{y z}+\sigma_{z} u_{x y}\right]\right\},
\end{gathered}
$$

where $\Xi_{c}$ is the intraband constant of the deformation potential responsible for the dominant (spin-independent) part of the electron-phonon interaction, and $\xi$ is the coefficient given by

$$
\xi=\frac{i \hbar P_{c v}}{3 m_{0}} \frac{\Delta_{s o}}{E_{g}\left(E_{g}+\Delta_{s o}\right)} .
$$

In deriving Eq. (44), we have expressed the strain tensor components in the primed axes via those in the QW coordinate system $x y z$ by using the equalities $u_{x^{\prime} y^{\prime}}=\left(u_{z z}-\right.$ $\left.u_{x x}\right) / 2, u_{x^{\prime} z^{\prime}}=-\left(u_{x y}+u_{y z}\right) / \sqrt{2}, u_{y^{\prime} z^{\prime}}=\left(u_{x y}-u_{y z}\right) / \sqrt{2}$.

The probability of electron scattering is determined by squared matrix elements of the electron-phonon interaction (4). The dominant contribution to spin-dependent asymmetry of the electron scattering in quantum wells is given by terms proportional to the $u_{z z}$ component of the phonon-induced strain tensor. This is because the strain tensor components depend on the wave vector and polarization of the phonon involved, and the in-plane component of the phonon wave vector $q_{\|}=\left|\mathbf{k}-\mathbf{k}^{\prime}\right|$ is typically much smaller than the out-of-plane component $q_{z} \sim \pi / a$, where $a$ is the QW width. Thus, the principle contribution to the scattering asymmetry in (110)-grown QW structures is proportional to $\sigma_{z}\left(k_{x}+k_{x}^{\prime}\right)$. This term is taken into account in calculations which follow. We note that $\mathbf{k}$-linear terms in the matrix elements of the electron-phonon interaction can also be obtained in the second order in the $\mathbf{k} \cdot \mathbf{p}$ theory, as was done in Ref. [10] for the electron scattering by charge impurities. However, these terms lead to no essential contribution to asymmetry of the electron scattering by phonons nor the spin orientation caused by the energy relaxation processes.

As is shown in Section III, spin-dependent asymmetry of the scattering processes followed by the precession of electron spins in the effective magnetic field leads to spin orientation of the electron gas. We assume that the spin relaxation time of carriers is much longer than the thermalization time controlled by electron-electron collisions 
which is in turn much longer than the momentum relaxation time $\tau_{p}$, and $\Omega_{\mathbf{k}} \tau_{p} \ll 1$. Then, the spin generation rate is given by [4]

$$
\dot{\mathbf{S}}=\sum_{\mathbf{k}} \tau_{p}\left[\boldsymbol{\Omega}_{\mathbf{k}} \times \mathbf{g}_{\mathbf{k}}\right]
$$

where $\mathbf{g}_{\mathbf{k}}=\operatorname{Tr}[\sigma G(\mathbf{k})] / 2, G(\mathbf{k})$ is the spin matrix describing the carrier redistribution in $\mathbf{k}$-space due to the scattering by phonons. Components of the matrix $G(\mathbf{k})$ have the form (see, e.g., Ref. [9] )

$$
\begin{gathered}
G_{s s^{\prime}}(\mathbf{k})=\frac{2 \pi}{\hbar} \sum_{s 1, \mathbf{k} 1} \sum_{\mathbf{q}, \pm} \times \\
\left\{V_{s \mathbf{k}, s 1 \mathbf{k} 1}^{( \pm)} V_{s^{\prime} \mathbf{k}, s 1 \mathbf{k} 1}^{( \pm) *} f\left(\varepsilon_{\mathbf{k} 1}\right)\left[1-f\left(\varepsilon_{\mathbf{k}}\right)\right] \delta\left(\varepsilon_{\mathbf{k}}-\varepsilon_{\mathbf{k} 1} \pm \hbar \omega_{\mathbf{q}}\right)\right. \\
\left.-V_{s 1 \mathbf{k} 1, s \mathbf{k}}^{( \pm) *} V_{s 1 \mathbf{k} 1, s^{\prime} \mathbf{k}}^{( \pm)} f\left(\varepsilon_{\mathbf{k}}\right)\left[1-f\left(\varepsilon_{\mathbf{k} 1}\right)\right] \delta\left(\varepsilon_{\mathbf{k} 1}-\varepsilon_{\mathbf{k}} \pm \hbar \omega_{\mathbf{q}}\right)\right\}
\end{gathered}
$$

where $V_{s \mathbf{k}, s 1 \mathbf{k} 1}^{( \pm)}$is the matrix element of the electron scattering assisted by emission $(+)$ or absorption $(-)$ of a phonon, $f\left(\varepsilon_{\mathbf{k}}\right)$ is the distribution function of carriers, $\varepsilon_{\mathbf{k}}=\hbar^{2} k^{2} /\left(2 m^{*}\right)$ is the kinetic energy, and $\omega_{\mathbf{q}}$ is the phonon frequency. Note that spin-orbit splitting of the energy spectrum is neglected in Eq. (17).

In (110)-grown QW structures, components of the Larmor frequency of the effective magnetic field have the form

$$
\boldsymbol{\Omega}_{\mathbf{k}}=\frac{2}{\hbar}\left(\gamma_{x y} k_{y}, \gamma_{y x} k_{x}, \gamma_{z x} k_{x}\right)
$$

The parameter $\gamma_{z x}$ is caused here by the lack of an inversion center in the host crystal, while $\gamma_{x y}$ and $\gamma_{y x}$ are non-zero due to the QW asymmetry only. We assume that electrons obey the Boltzmann statistics and the lattice temperature is not very low, $k_{B} T_{0} \gg \hbar \omega_{\mathbf{q}}$. Then, one derives

$$
\dot{S}_{x}=-\tau_{p} \gamma_{y x} \frac{\Xi_{c} \Xi_{c v}}{2 \rho} \frac{\xi m^{* 2}}{\hbar^{4}} \frac{\Delta T}{T_{e}} N_{e} \times \int_{-\infty}^{+\infty}\left[\frac{d \varphi^{2}(z)}{d z}\right]^{2} d z
$$

where $\rho$ is the crystal density, $N_{e}=2 \sum_{\mathbf{k}} f\left(\varepsilon_{\mathbf{k}}\right)$ is the two-dimensional electron concentration, and $\varphi(z)$ is the function of size quantization.

In the steady state regime, when the electron temperature $T_{e}$ and the lattice temperature $T_{0}$ are held constant, the spin density $S_{x}$ is determined by balance between the spin generation and relaxation processes; $S_{x}=\dot{S}_{x} T_{x}$, where $T_{x}$ is the spin relaxation time. In (001)-grown QWs, the time $T_{x}$ is given by [1]

$$
T_{x}^{-1}=-\int_{0}^{\infty} \frac{\tau_{p}}{f(0)} \frac{d f\left(\varepsilon_{\mathbf{k}}\right)}{d \varepsilon_{\mathbf{k}}}\left(\left\langle\Omega_{\mathbf{k}, y}^{2}\right\rangle+\left\langle\Omega_{\mathbf{k}, z}^{2}\right\rangle\right) d \varepsilon_{\mathbf{k}},
$$

where the angle brackets mean averaging over directions of the wave vector. For the Boltzmann distribution, Eq. (10) assumes the form

$$
T_{x}^{-1}=\frac{4 m^{*} \tau_{p}}{\hbar^{4}}\left(\gamma_{y x}^{2}+\gamma_{z x}^{2}\right) k_{B} T_{e} .
$$

Finally, we obtain for the steady spin density

$$
S_{x}=-\frac{m^{*} \xi}{8 \rho} \frac{\gamma_{y x} \Xi_{c} \Xi_{c v}}{\gamma_{y x}^{2}+\gamma_{z x}^{2}} \frac{\Delta T N_{e}}{k_{B} T_{e}^{2}} \times \int_{-\infty}^{+\infty}\left[\frac{d \varphi^{2}(z)}{d z}\right]^{2} d z
$$

The estimation for the average electron spin gives $S_{x} / N_{e} \sim 10^{-5}$ for the electron temperature $T_{e}=100 \mathrm{~K}$, the ratio $\Delta T / T_{e} \approx 1$, the quantum well width $a=100 \AA$, and band parameters $m^{*}=0.07 m_{0}, \xi=0.4 \AA, \gamma / \hbar=$ $10^{5} \mathrm{~cm} / \mathrm{s}, \Xi_{c}=-8 \mathrm{eV}, \Xi_{c v}=3 \mathrm{eV}$ [1] corresponding to GaAs-based QW structures. Thus, for the carrier density $N_{e}=10^{11} \mathrm{~cm}^{-2}$, the spin density $S_{x}$ is of the order of $10^{6} \mathrm{~cm}^{-2}$ which is well above the experimental resolution. We also note that the modest estimated value of the spin polarization is due to the fact that the energy relaxation by acoustic phonons is ineffective. The spin polarization can considerably increase if optical phonons are involved in the energy relaxation processes.

Acknowledgments. This work was supported by the RFFI, programs of the RAS, and the President Grant for young scientists.
[1] Optical Orientation, edited by F. Meier and B.P. Zakharchenya (Elsevier Science, Amsterdam, 1984).

[2] M.I. D'yakonov and V.I. Perel', Possibility of orienting electron spins with current, Pis'ma Zh. Eksp. Teor. Fiz. 13, 657 (1971) [JETP Lett. 13, 467 (1971)].

[3] V.I. Perel', S.A. Tarasenko, I.N. Yassievich, S.D. Ganichev, V.V. Bel'kov, and W. Prettl, Spindependent tunneling through a symmetric semiconductor barrier, Phys. Rev. B 67, 201304 (2003).

[4] S.A. Tarasenko, Optical orientation of electron spins by linearly polarized light, Phys. Rev. B 72, 113302 (2005); Spin orientation of a two-dimensional electron gas by a high-frequency electric field, Phys. Rev. B 73, 115317 (2006).

[5] E.L. Ivchenko and G.E. Pikus, Optical orientation of free carriers spins and photogalvanic effects in gyrotropic crystals, Izv. Akad. Nauk SSSR, Ser. Fiz. 47, 2369 (1983) [Bull. Acad. Sci. USSR, Phys. Ser. 47, 81 (1983)].

[6] S.D. Ganichev, V.V. Bel'kov, S.A. Tarasenko, S.N. Danilov, S. Giglberger, Ch. Hoffmann, E.L. Ivchenko, D. Weiss, W. Wegscheider, C. Gerl, D. Schuh, J. Stahl, J. De Boeck, G. Borghs, and W. Prettl, Zero-bias spin separation, Nature Physics 2, 609 (2006). 
[7] M.I. D'yakonov and V.I. Perel', Spin relaxation of conduction electrons in noncentrosymmetric semiconductors, Fiz. Tverd. Tela (Leningrad) 13, 3581 (1971) [Sov. Phys. Solid State 13, 3023 (1971)].

[8] Yu.A. Bychkov and E.I. Rashba, Properties of a 2D electron gas with lifted spectral degeneracy, Pis'ma Zh. Eksp. Teor. Fiz. 39, 66 (1984) [JETP Lett. 39, 78 (1984)].

[9] E.L. Ivchenko and S.A. Tarasenko, Monopolar optical orientation of electron spins in bulk semiconductors and heterostructures, Zh. Eksp. Teor. Fiz. 126, 476 (2004)
[JETP 99, 379 (2004)].

[10] N.S. Averkiev, L.E. Golub, and M. Willander, Spin relaxation anisotropy in two-dimensional semiconductor systems, J. Phys.: Condens. Matter 14, R271 (2002).

[11] M.I. D'yakonov and V.Yu. Kachorovskii, Spin relaxation of two-dimensional electrons in noncentrosymmetric semiconductors, Fiz. Tekh. Poluprovodn. (Leningrad) 20, 178 (1986) [Sov. Phys. Semicond. 20, 110 (1986)]. 